\section{$\underset{\substack{\text { hommes } \\ \text { \& migrations }}}{ }$}

\section{Hommes \& migrations}

Revue française de référence sur les dynamiques

migratoires

\section{$1321 \mid 2018$}

Les mots de l'exil dans l'Europe du XIXe siècle

\title{
L'exil carliste espagnol dans le sud de la France des années 1870 :
}

Entre catégorisations du réfugié et protestations populaires

\section{Alexandre Dupont}

\section{(2) OpenEdition}

Journals

Édition électronique

URL : http://journals.openedition.org/hommesmigrations/4335

DOI : ERREUR PDO dans /localdata/www-bin/Core/Core/Db/Db.class.php L.34 : SQLSTATE[HY000]

[2006] MySQL server has gone away

ISSN : 2262-3353

Éditeur

Musée national de l'histoire de l'immigration

Édition imprimée

Date de publication : 1 avril 2018

Pagination : 93-100

ISBN : 978-2-919040-41-4

ISSN : $1142-852 X$

\section{Référence électronique}

Alexandre Dupont, «L'exil carliste espagnol dans le sud de la France des années 1870 : », Hommes \& migrations [En ligne], 1321 | 2018, mis en ligne le 01 avril 2020, consulté le 08 janvier 2021. URL

http://journals.openedition.org/hommesmigrations/4335; DOl : https://doi.org/10.4000/

hommesmigrations.4335 


\title{
L'EXIL CARLISTE ESPAGNOL DANS LE SUD DE LA FRANCE DES ANNÉES 1870 : ENTRE CATÉGORISATIONS DU RÉFUGIÉ ET PROTESTATIONS POPULAIRES
}

Par ALEXANDRE DUPONT, maître de conférences en histoire contemporaine, université de Strasbourg.

\author{
Dans les années 1870, marquées par d'importants mouvements \\ migratoires de I'Espagne vers la France, la gestion \\ des ressortissants espagnols devient une affaire épineuse pour \\ I'État français. La France sert à la fois de refuge à de nombreux \\ Espagnols mais aussi de base arrière à l'insurrection carliste qui \\ tente de renverser le gouvernement de Madrid. À l'échelle de \\ la frontière entre les deux pays se joue une situation complexe \\ où les parcours individuels des Espagnols soulèvent un enjeu \\ à la fois diplomatique et politique, entre gestion juridique des \\ réfugiés et nécessité de préserver la paix des populations \\ frontalières.
}

À la mi-août 1870, l'émoi s'empare d'Urepel, un village français d'environ 900 habitants situé à la frontière avec la Navarre, dans la vallée des Aldudes. Les villages espagnols voisins menacent, en effet, d'incendier Urepel si les habitants s'obstinent à héberger sept exilés carlistes. Partisans d'un retour à la monarchie catholique traditionnelle, les carlistes espagnols tentent depuis la Révolution de septembre 1868 de reprendre le pouvoir. La France de la fin du Second Empire (1852-1870) leur sert de base arrière où ils peuvent aisément se réfugier. En 1872, lorsqu'ils déclenchent une guerre civile contre le gouvernement de Madrid, ce refuge français leur est d'une grande utilité, malgré la répression que la Troisième République installée en 1870 exerce contre eux.
Inquiets, les habitants d'Urepel en appellent aux autorités et, en particulier, au sous-préfet de Mauléon qui prend l'affaire en main et envoie sur place dès le 15 août la brigade de gendarmerie postée au village d'Irissarry. Conseillés par les autorités locales et les gendarmes, les Urepeldars souhaitent envoyer les carlistes vers Mauléon. Le sous-préfet est bien embarrassé par ces « carlistes établis » dont il ne sait pas quoi faire. Détenus au dépôt de sûreté d'Urepel, ceux-ci demandent à être mis en liberté pour se rendre auprès du consul espagnol de Bayonne, demander l'amnistie et rentrer en Espagne. La solution semble enchanter le sous-préfet ${ }^{1}$.

Cette affaire, en apparence anecdotique, souligne l'extrême difficulté que pose la gestion quotidienne des 
étrangers à la frontière entre France et Espagne dans les années 1870. En 1870, la guerre carliste, qui touche les provinces du nord de l'Espagne entre 1872 et 1876, n'a pas encore commencé, mais une conflictualité de basse intensité est déjà à l'œuvre et conduit nombre de carlistes à se réfugier en France après tel ou tel coup de main ${ }^{2}$. Près de la frontière, ils se mêlent à d'autres Espagnols présents de plus ou moins longue date et pour les motifs les plus divers : difficile pour les autorités de définir qui est qui et de prendre des décisions administratives en conséquence. L'embarras du sous-préfet face au cas de ces hommes, établis depuis un certain temps et que leur départ d'Urepel plonge dans la

En 1870, la guerre carliste,

qui touche les provinces

du nord de l'Espagne

entre 1872 et 1876 , n'a pas

encore commencé, mais

une conflictualité de basse

intensité est déjà à l'œuvre et conduit nombre de carlistes à se réfugier en France après tel

ou tel coup de main. misère, est révélateur : faut-il venir en aide à des réfugiés, victimes d'un coup du sort, ou sévir contre des légitimistes espagnols désireux de troubler la paix du pays voisin? On comprend qu'il se soit empressé de saisir la porte de sortie offerte par les carlistes qui demandent à rentrer chez eux. Et ce d'autant plus que les autorités doivent aussi tenir compte des réactions et des revendications des communautés locales. On est en droit de supposer que les voisins d'Urepel n'ont proféré leurs menaces qu'après plusieurs épisodes de conflits, où les habitants d'Urepel ont refusé d'expulser les carlistes de leur village.

Cet article se propose d'interroger la construction des catégories administratives de gestion des étrangers dans la France du XIX ${ }^{e}$ siècle en partant d'une analyse micro-historique ${ }^{3}$. Létude portera sur les exilés carlistes espagnols en France pendant la guerre civile de 1872-1876, car le contexte guerrier a des répercussions importantes sur la pratique des autorités françaises. Le choix d'une échelle locale et frontalière vise à mieux mettre en exergue le rôle des classes populaires dans la construction de ces catégories administratives, par leur capacité à s'opposer à des processus de définition abstraits imposés d'en haut, au nom d'une prise en compte des enjeux locaux et des situations particulières.

\section{Identifier les carlistes}

Lorsquéclate la guerre carliste de 1872-1876, les autorités françaises ne sont pas prises au dépourvu par l'entrée d'exilés espagnols sur le sol français. Depuis le début du siècle, plusieurs éléments ont conduit à une sédimentation de règles et de pratiques à adopter face à ces traversées de la frontière. Trois processus ont revêtu une importance particulière.

D'abord, l'Espagne devient, dès les années 1810, l'un des principaux pourvoyeurs de réfugiés politiques en France. L'exil des joséphins, partisans de Joseph Bonaparte, en 1813 est rapidement suivi par celui des libéraux après la restauration de 1814. Cet épisode ouvre un long cycle de migrations politiques depuis l'Espagne qui oblige les autorités françaises à gérer ces flux ${ }^{4}$. Au sein de ces exilés espagnols, les contre-révolutionnaires prennent toute leur place, d'abord en 1820 après la prise de pouvoir par les libéraux, mais surtout en 18391840. L'échec des carlistes à mettre sur le trône le prétendant don Carlos, frère du défunt roi Ferdinand VII, après sept ans de combat, conduit alors au passage en France de près de 40000 soldats, une migration politique massive face à laquelle l'État s'organise ${ }^{5}$.

C'est un deuxième faisceau qui explique la formation d'un cadre de gestion des déplacés en France 
au XIX siècle : à partir de la monarchie de Juillet, le pays devient l'une des terres d'accueil privilégiées par les exilés. Les autorités françaises adoptent peu à peu la pratique de l'internement. Elle concerne réfugiés civils et militaires, qui sont assignés à résidence dans des villes qu'ils ne peuvent quitter sans autorisation et où ils reçoivent des subsides de la part du gouvernement ${ }^{6}$. Ce cadre général établi par la loi de 1832 est ensuite adapté aux différents groupes d'exilés : les carlistes se voient ainsi interdire la résidence près de la frontière et dans les départements de l'Ouest, où l'on craint une collusion avec les légitimistes?

Enfin, le contexte politique espagnol après la révolution de 1868, qui renverse la reine Isabelle II, conduit à une recrudescence des exils politiques depuis l'Espagne, en particulier parmi les carlistes. Dès le mois d'octobre 1868, quelques semaines après la révolution, les autorités de Gérone demandent ainsi aux autorités françaises d'interner les carlistes présents sur la frontière catalane ${ }^{8}$. Les mesures à l'encontre des légitimistes espagnols qui passent en France ne datent donc pas du soulèvement carliste d'avril 1872. Le journaliste carliste Julio Nombela rappelle par exemple, dans un ouvrage de 1871, la grande campagne d'internement mise en place par les autorités françaises au printemps $1870^{9}$.

\section{Principes et pratiques de l'internement}

Le déclenchement de la guerre carliste met le gouvernement français face au défi d'une traversée massive et régulière de la frontière par des Espagnols des deux camps, que ce soit pour échapper aux combats, pour poursuivre l'adversaire ou, dans le cas des carlistes, parce qu'ils ont fait des départements frontaliers une arrière-cour qui leur assure une base logistique dans le conflit. Deux impératifs se présentent donc au gouvernement français : faire respecter l'intégrité du territoire et empêcher une propagation du conflit sur le sol national ; sévir contre le passage de carlistes en France, au nom des relations diplomatiques existantes avec Madrid.

À partir de ces données, les autorités édictent dans les premiers jours du soulèvement des règles précises. Des instructions sont envoyées aux chefs des détachements militaires sur la frontière pour contrôler les déplacements et les entrées en France. C'est ainsi que le général Lefort, qui commande une division du XVIII corps d'armée chargée de garder la frontière dans les Basses-Pyrénées, adresse à ses subordonnés une liste d'indications très minutieuses le 23 avril 1872, deux jours à peine après l'appel aux armes de don Carlos $^{10}$.

Ces indications montrent que les
Lorsqu'éclate la guerre carliste de 1872-1876, les autorités françaises ne sont pas prises au dépourvu par l'entrée d'exilés espagnols sur le sol français. très tôt le problème de la catégorisation des Espagnols qui traversent la frontière et tâchent d'apporter une réponse adéquate en fonction de leur statut. Les trois premiers articles réglementent l'entrée d'agents du gouvernement espagnol sur le sol français et vice-versa. Les militaires des deux pays se voient interdire de traverser la frontière, sauf autorisation expresse.

Les deux articles suivants visent, d'une part, les déserteurs des deux armées opposées et, d'autre part, les partisans carlistes, désignés comme des "réfugiés politiques » : les uns comme les autres ont vocation à être désarmés et présentés aux autorités civiles qui doivent décider de leur internement. Dans les articles 8 et 9, c'est le sort des groupes de belligérants passés en France qui est examiné. Dans le cas des troupes gouvernementales, le choix doit leur être donné entre se retirer en Espagne ou se soumettre à l'internement. Enfin, les articles 10 et 11 évoquent le cas des civils. Ceux 
qui fuient les combats doivent ainsi être reçus avec égard et ne pas être internés. Les frontaliers traversant la frontière pour leurs affaires ne relèvent pas des compétences de l'armée, mais des autorités civiles.

Ainsi, les autorités françaises s'efforcent de procéder à une double série de définitions. D'une part, elles tâchent de faire la différence, parmi les Espagnols, entre passages individuels et passages groupés, passages de carlistes, de soldats du gouvernement, de civils et de réfugiés; dans le temps de la guerre civile, l'internement vise donc plus particulièrement les carlistes en armes, même s'il vise Le déclenchement ponctuellement des civils identide la guerre carliste met le gouvernement français face au défi d'une traversée massive et régulière de la frontière par des Espagnols des deux camps. fiés comme agents carlistes. D'autre part, se met en place une répartition des responsabilités et des ressorts d'action entre les différents représentants de l'État, et en particulier entre militaires et civils.

Fortes de ce cadre juridico-administratif, les autorités françaises s'appliquent donc à établir des distinctions tout au long de la guerre, et à agir en conséquence. Du côté de la Catalogne, où les combats font rage en 1873 et 1874 autour de Puigcerdà et La Seo de Urgel, de nombreux soldats gouvernementaux passent ainsi la frontière au cours de ces années. Peu à peu, une procédure se met en place qui se standardise au fil du temps : après leur passage de la frontière, soldats et sous-officiers sont désarmés et envoyés dans des casernes de la région, pendant que les officiers conservent leurs armes et sont logés dans de meilleures conditions. Assez rapidement, ces hommes sont ensuite renvoyés en Espagne via le port de Sète ${ }^{11}$.

Les nombreux combats qui se déroulent autour de la ville frontalière de Puigcerdà, en Cerdagne espagnole, provoquent d'importants mouvements de populations à travers la frontière. Là encore, la politique d'accueil de ces réfugiés qui fuient les combats est mise en œuvre. Le commissaire de Bourg-Madame, Joseph Agel, laisse un récit saisissant de l'un de ces mouvements de population en juillet 1873 : «Les déménagements de Puigcerda dans les villages français limitrophes ont continué toute la soirée: (...) on avait mis en ceuvre tous les moyens de transport ; cétait une panique générale. (...) De ce côté de la frontière, les fuyards ou réfugiés trouvent la plus large hospitalité $e^{12}$."

Ceux désignés comme des exilés, c'est-à-dire comme des individus ayant quitté leur pays pour des raisons politiques ${ }^{13}$, font pour leur part l'objet de mesures d'internement. Dans les PyrénéesOrientales, ce sont ainsi plus de 600 carlistes qui sont internés par les sous-préfets et le préfet entre 1872 et 1874 et pour lesquels on dispose d'états nominatifs mettant en valeur la rationalisation de la procédure ${ }^{14}$. Dans ces états nominatifs, les carlistes sont de loin les plus nombreux parmi les exilés internés. Toutefois, ils sont accompagnés par un certain nombre de déserteurs et de républicains. Au sein même de la catégorie des exilés, les autorités s'efforcent donc de faire la distinction entre différentes «familles », les déserteurs étant assimilés à des opposants au pouvoir.

Ainsi, l'État semble être parvenu à mettre en place un dispositif de surveillance et de contrôle de la frontière lui permettant de «trier » les Espagnols passant en France en fonction de leur statut, et de leur appliquer telle ou telle mesure en fonction de ce statut. Si ce système connaît d'indéniables résultats, il y a toutefois loin de cet appareil théorique à la pratique inscrite dans un contexte social dont il faut tenir compte.

\section{Les politiques étatiques à l'épreuve du terrain}

Le dispositif mis en place par l'État pour identifier, contrôler et interner les exilés carlistes en France 
atteint très rapidement ses limites sur le terrain. Plusieurs raisons expliquent cette impuissance relative de l'État. La première est que la frontière est trop étendue pour que les autorités puissent prétendre la contrôler entièrement, et donc arrêter tous les individus qui la traversent. Dans un mémoire rédigé en 1874, au plus fort de la crise diplomatique entre Paris et Madrid au sujet du carlisme, le baron Pierlot rappelle cette réalité : «Si des individus sont parvenus à déjouer les dispositions prises, on se l'explique aisément lorsqu'on réfléchit à l'étendue et à la configuration de la ligne que nous avons à garder. Pour prévenir toute infraction de ce genre, il ne faudrait pas moins qu'empêcher tout rapport, c'est-à-dire suspendre en quelque sorte la vie même entre les frontaliers ${ }^{15}$. »

À cette première difficulté ayant trait aux moyens dont dispose l'État pour contrôler les déplacements, s'ajoute un problème de fond relatif à l'identification des carlistes. Bien sûr, les autorités n'ont pas de doutes lorsqu'il s'agit d'une troupe de soldats carlistes qui passe en France. Mais, dans le cas d'exilés carlistes civils, deux séries de difficultés se posent. La première a trait à l'identification des personnes. En effet, la convention consulaire franco-espagnole de 1862 accorde la libre circulation aux ressortissants des deux pays ${ }^{16}$. Dès lors, pour contrôler l'identité et l'appartenance politique des personnes, les autorités françaises s'emploient dès le début de la guerre à imposer à nouveau l'usage du passeport à la frontière, comme cela avait été le cas lors de précédents épisodes de troubles en Espagne ${ }^{17}$.

La deuxième série de difficultés est liée à la présence massive des Espagnols dans les départements frontaliers. Au mois de décembre 1872, le général Lefort signale au ministre de la Guerre que la répression contre les exilés carlistes restera impuissante tant qu'on laissera les Espagnols résider librement dans les départements frontaliers ${ }^{18}$. Ce point de vue des autorités militaires se heurte dès lété 1872 à l'opposition des autorités civiles et notamment des préfets. Au mois de juin, le marquis de Nadaillac, préfet des

Basses-Pyrénées, demande ainsi que l'on se limite à interner les exilés carlistes ayant pris part à l'insurrection, ce que le gouvernement accepte ${ }^{19}$. Le renforcement de l'insurrection conduit la France, au début de l'année 1873, à interdire aux Espagnols de résider dans un certain nombre de communes de la frontière ${ }^{20}$ et à interner tous les Espagnols des départements frontaliers ne disposant pas d'une immatriculation consulaire. Ces mesures, demandées par l'armée, sont encore une fois critiquées par Nadaillac ${ }^{21}$.

Si cette attitude vaudra au préfet une réputation durable de partisan de l'insurrection, elle prouve à la fois la difficulté d'identifier les exilés dans un contexte social où la présence espagnole est forte, ainsi que les divisions entre différentes instances porteuses d'intérêts et de préoccupations différents. De fait, l'attitude de Nadaillac, plus que d'un soutien à don Carlos, est révélatrice d'un souci de maintenir le calme au sein d'une population qui s'invite dans le débat sur les exilés - préoccupation qui touche moins les responsables militaires à la fron-

Dans les Pyrénées-Orientales, ce sont ainsi plus de 600 carlistes qui sont internés par les sous-préfets et le préfet entre 1872 et 1874 et pour lesquels on dispose d'états nominatifs mettant en valeur la rationalisation de la procédure

tière, ce qui explique leur plus grande sévérité. Au niveau gouvernemental, de telles dissensions se retrouvent dans les discussions entre le ministre de la Guerre et le ministre de l'Intérieur, qui doivent aussi tenir compte du point de vue de leur collègue des Affaires étrangères ; pour autant, cette communication intra-gouvernementale débouche la plupart du temps sur une résolution des conflits internes à l'appareil d’État français.

\section{Contestations populaires}

La complexité de la situation des Espagnols à la frontière, entre carlistes exilés, travailleurs 
saisonniers, agents de don Carlos et Espagnols installés de longue date, complique l'intervention des autorités et l'identification des exilés jouant un rôle politique actif. Dans le même temps, les modalités d'action des pouvoirs publics provoquent des réactions au sein de la population, réactions qui influencent en retour cette action.

Les protestations contre les mesures d'internement prises contre des exilés procèdent en partie de partisans français de la cause carliste, ces derniers étant particulièrement nombreux dans les départements frontaliers ${ }^{22}$. Dans les Pyrénées-Orientales, le journal Le Drapeau français, organe de presse de l'état-major carliste en Catalogne, va jusqu'à mettre sur pied un «Comité de consultation et de protection pour les Espagnols en résidence dans le département » et assure qu' " au cas où ils seraient inquiétés le comité se met à leur disposition pour les recours judiciaires, quills pourraient invoquer le cas échéant ${ }^{23}$ ".

En somme, une première modalité d'intervention des citoyens dans les processus de contrôle des exilés est le fait d'une solidarité politique transnationale qui les pousse à dénier à l'État le droit de réprimer les exilés politiques. Cette modalité d'action est évoquée en 1873 par le consul d'Espagne à Perpignan, dans une lettre où il se plaint de l'hostilité des villages de la frontière à l'action des représentants espagnols. Ce n'est pourtant que la troisième raison qu'il invoque, après la sympathie naturelle des populations envers les réfugiés politiques et, surtout, après la prééminence des relations sociales sur les considérations politiques: "Pendant de nombreuses années, y [dans les villages de la frontière] ont résidé des émigrés carlistes qui ont tissé des liens d'amitié et même de parenté24.

Le consul indique ainsi que les populations frontalières se mobilisent notamment du fait de l'insertion des exilés carlistes dans le tissu social local. Les sources reflètent effectivement ces très nombreuses mobilisations en faveur de tel ou tel exilé. À l'été
1875, la ville de Céret s'investit ainsi en faveur de Francisco Manresa de Bassols, un réfugié carliste installé en France depuis 1835 dont l'internement est demandé par le consul de Perpignan. Lémotion provoquée est vive en raison de l'ancienneté de la présence de Manresa en France, des liens familiaux qu'il a dans la région depuis son mariage avec une Française et de son éloignement des questions politiques depuis des années.

L'unanimité est telle que la pétition en sa faveur est signée par des radicaux de Céret, et que le préfet des Pyrénées-Orientales appuie la demande de clémence en faveur de cet exilé qui, par son insertion dans la sociabilité locale, n'en est plus vraiment $u^{25}$. Son avocat l'affirme dans une lettre au préfet : «M. de Manresa n'est pas réfugié en France, il est chez lui ; M. de Manresa n'est pas un étranger, il peut exercer tous les droits civils réglés par le code français; $M$. de Manresa n'est pas près de la frontière, il est dans ses propriétés, à la tête de sa famille (... ${ }^{26}$. "

C'est donc la qualité même d'étranger et d'exilé politique que ces mobilisations populaires contribuent à interroger et à remettre en question. Côté basque, un agent espagnol faisant son rapport au gouverneur civil du Guipúzcoa s'exaspère en juin 1874 : "Je n'en finirais jamais s'il me fallait rapporter des actes fréquents de protection que les habitants et les autorités locales de la frontière française prodiguent aux $\operatorname{carlistes}^{27}$ ", associant par là même cette mobilisation en faveur des exilés à un soutien au carlisme. C'est parfois le cas, mais il ne s'agit pas d'une règle générale : ce soutien est bien plus souvent le produit de sociabilités transfrontalières anciennes entre communautés paysannes des deux côtés de la frontière, ces communautés paysannes offrant au carlisme une bonne partie de ses recrues dans le Pays basque et la Catalogne espagnols. Le soutien des autorités locales à un certain nombre de ces initiatives populaires invite par ailleurs à interroger à nouveaux frais ces protestations.

22. Voir aussi Alexandre Dupont, "Comprendre la dissidence : les Basques et les Catalans de France, soutiens du carlisme? ", in Bulletin des doctorants et jeunes chercheurs du Centre d'histoire du XIXe siècle, nº 4-5, 2016, p. 9. 23. Le Drapeau français, 15 juin 1873.24 . AHN,

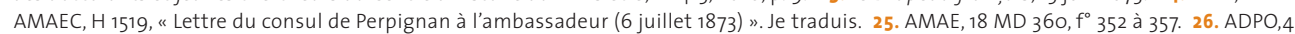
M 467, "Mémoire de Albert Tolza, avocat, au préfet (juillet 1875) ». 27. AN, F7 12 578, "Rapport au gouverneur civil de la province de Guipúzcoa (25 juin 1874) ». 


\section{Les fonctionnaires locaux, une interface entre l'État et le monde social}

Il existe une troisième modalité d'intervention de la population dans la catégorisation des étrangers, qui consiste à contester les velléités du gouvernement français de renforcer son emprise sur la région frontalière par le contrôle des déplacements ${ }^{28}$. Cette tentative s'est traduite par l'introduction du passeport pour les Espagnols souhaitant se rendre en France et par l'obligation pour ces mêmes Espagnols de se faire immatriculer auprès de leurs consulats, sous peine d'expulsion ou d'internement. Une mesure extrême a consisté à interdire à tous les Espagnols la résidence dans certaines villes de la frontière. À toutes ces demandes présentées par les autorités militaires, le préfet Nadaillac a tenté de s'opposer. Les raisons de cette opposition, on l'a dit, procèdent pour une bonne part de son souci de maintenir l'ordre public dans son département.

C'est principalement contre ce type de mesures que s'insurgent les populations frontalières au cours de la guerre. La généralisation de ces mesures à tous les Espagnols présents dans les départements frontaliers, tout comme la remise en cause de circulations transfrontalières traditionnelles au nom de la lutte contre le carlisme, font l'objet d'une réprobation générale de la part des habitants de la frontière. Les autorités locales doivent s'interposer entre leurs administrés et le gouvernement, et se présenter comme médiateurs auprès de ce dernier afin qu'il assouplisse des positions qui se justifient en termes de rationalité et d'efficacité administratives, mais constituent des facteurs de troubles dans la zone frontalière ${ }^{29}$.

Dans les Basses-Pyrénées, dès 1872, une mobilisation populaire parvient à infléchir par deux fois la politique du gouvernement. Celui-ci avait décrété, en avril, l'internement de tous les
Espagnols résidant dans le département. Cela provoque la réaction quasi-immédiate des habitants de la ville de Saint-Jean-de-Luz qui, par la voix des autorités municipales, font savoir au préfet leur opposition résolue à une mesure à la fois extrême et injuste. Deux séries d'arguments sont mobilisés : d'une part, il est injuste d'assimiler tous les Espagnols du département à des factieux ; d'autre part, une telle mesure menace gravement la prospérité économique de la région frontalière, dépendante à de multiples titres de ses relations avec l'Espagne. Avec les mêmes arguments, Nadaillac parvient à limiter la répression à ceux qui ont participé au mouvement ou l'ont soutenu : il reprend la distinction entre réfugié, exilé politique et simple civil ${ }^{30}$.

Quelques mois plus tard, La complexité de la situation Nadaillac, s'appuyant sur l'échec des Espagnols à la frontière, du soulèvement dans les pro- entre carlistes exilés, vinces basques dès le mois de travailleurs saisonniers, mai ${ }^{31}$, obtient du gouvernement agents de don Carlos et que l'internement ne concerne Espagnols installés de plus tous les exilés politiques, Iongue date, complique mais seulement ceux ayant par- l'intervention des autorités ticipé au soulèvement ou et l'identification des exilés conspirant pour le rallumer, ce jouant un rôle politique actif. qui constitue l'adoption d'un nouveau critère de distinction, au sein des exilés eux-mêmes. Cette décision du ministre s'appuie sur l'envoi par Nadaillac «[d'] une pétition formée par un grand nombre d'habitants et de commerçants de cette ville, à l'effet d'obtenir des adoucissements à la rigueur des mesures prises au début de l'insurrection carliste contre les résidents et voyageurs Espagnols, mesures qui auraient pour effet d'eloigner de Bayonne et des villes voisines les familles espagnoles qui passent habituellement une partie de l'été dans les départements Pyrénéens ${ }^{32} »$. Par deux fois, en quelques mois, la mobilisation des populations frontalières des Basses-Pyrénées, s'appuyant sur des considérations socio-économiques, sera parvenue à infléchir la politique gouvernementale de traitement des 
exilés et plus largement des Espagnols, révélant in fine le caractère social de la construction de ces catégories du pouvoir.

\section{Conclusion}

Le traitement réservé par l'État aux carlistes exilés en France pendant la guerre carliste de 1872-1876 illustre ainsi un double processus contradictoire. D'une part, il souligne le développement par les services de l'administration et par les forces de l'ordre de catégories et d'outils de contrôle des personnes et des déplacements qui rendent la gestion des étrangers en France de plus en plus rationalisée et efficace, notamment grâce à une sédimentation de règlements et de pratiques. La capacité à distinguer entre réfugiés, exilés et simples civils, voire d'établir des sous-catégories au sein de cette nomenclature, ainsi que l'adaptation du dispositif à l'évolution de la situation, sont autant de signes du fait que la construction de l'État libéral est une réalité dans cette seconde moitié du XIXe siècle, réalité qui s'observe notamment dans sa capacité à contrôler les étrangers.
D'autre part, cependant, la pratique de ce contrôle des étrangers se révèle beaucoup plus hasardeuse. D'abord, il est bien difficile de faire correspondre un tel schéma théorique à un monde social frontalier où les identités des uns et des autres ne sont pas aussi claires. Ensuite, les autorités doivent prendre en compte les protestations de groupes d'acteurs des classes populaires mobilisés pour des raisons différentes: soutien transnational au carlisme, appui à des individus particulièrement bien insérés dans le tissu local, opposition à une remise en cause d'une sociabilité transfrontalière déterminante dans la vie économique des départements frontaliers. Ces mobilisations, relayées par des fonctionnaires locaux soucieux de ne pas déstabiliser les espaces de leur juridiction, sont parfois victorieuses et soulignent le fait que la construction des catégories d'étranger et d'exilé dans la France du XIX' siècle se déterminent aussi en fonction de ces mobilisations populaires. L'observation micro-historique de ces processus dans la zone frontalière permet en outre de mettre en valeur le rôle fondamental joué par les agents locaux de l'État dans la construction progressive et relativement pacifique de son contrôle sur son territoire, et en particulier sur ses frontières. 